\title{
Targets detection in sea clutter based on echo state network
}

\author{
Zhan $\mathrm{Xu}^{1, \mathrm{a}}$, Jianwei Wan ${ }^{1, \mathrm{~b}}$, Gang $\mathrm{Li}^{1, \mathrm{c}}$ and Fang Su${ }^{1, \mathrm{~d}}$ \\ ${ }^{1}$ School of Electronic Science and Engineering, National University of Defense Technology, \\ Changsha, 410073, People's Republic of China
}

aEmail: xuzhan121@163.com

Keywords: Target detection, sea clutter, echo state network.

\begin{abstract}
A novel method to predict the sea clutter time series and detect target embedded in sea clutter is presented. The method is actually a recurrent neural network called an echo state network (ESN). A recursive least squares (RLS) algorithm is used for updating the output weights of ESN. A set of time series from IPIX radar data is tested. Numerical experiments reveal that the proposed network shows higher prediction precision in pure sea clutter data. Moreover, the mean squared error (MSE) between real-life data and prediction value by ESN can be used to detect target effectively.
\end{abstract}

\section{Introduction}

Echo state network (ESN) is a novel recurrent neural network (RNN) architecture which was introduced by Herbert Jaeger for time series prediction [1-3]. The kernel part of ESN is a single reservoir with tens or hundreds of neurons that are randomly and sparsely interconnected. The degree of the sparseness is from $2 \%$ to $20 \%$. The reservoir is fixed, that is to say, the reservoir itself is not changed once the network is setup. During the training process of ESN, only the output connections are changed through offline linear regression or online methods, such as the recursive least square (RLS) [1-3]. ESN has dynamics characteristic and short memory function. The stability of ESN is better than the traditional networks, and can be successfully applied in chaotic and nonlinear dynamic systems modeling, identification and control [3-5]. We apply ESN to predict the sea clutter time series. The great mean squared error (MSE) differences between real-life sea clutter data and prediction value by ESN are available for target detection.

\section{ESN Model}

The general structure of an ESN with an N-neuron reservoir is shown in Fig.1. The number of input layer nodes is $\mathrm{K}$, and the number of output layer nodes is L. The update of the reservoir state is expressed by

$$
x(n)=f\left(W_{\text {in }} u(n)+W x(n-1)+W_{b} d(n-1)+\gamma(n)\right)
$$

where $\mathrm{f}$ is a sigmoidal activation function, $\mathrm{x}(\mathrm{n})$ is the internal state of the reservoir at time step $\mathrm{n}, \mathrm{u}(\mathrm{n})$ is the input vector at time step $\mathrm{n}, \mathrm{d}(\mathrm{n}-1)$ is the teacher signal (real-life data or test sample) at the previous time step, and $\gamma(\mathrm{n})$ is the current artificial noise vector inserted into the state update equation to ensure stability of the network. In general, the range for $\gamma(\mathrm{n})$ is from 0.0001 to 0.01 . The input weight $\mathrm{W}_{\text {in }}$ is an $\mathrm{N} \times \mathrm{K}$ matrix which reflects the connection from input nodes to the reservoir. The entries of this matrix are selected at random. The recurrent weight $\mathrm{W}$ is an $\mathrm{N} \times \mathrm{N}$ matrix which reflects the interconnection in the reservoir. This matrix is a sparse and random matrix. To ensure echo characteristic in ESN, the spectral radius $\rho(\mathrm{W})$ must be less than 1 . The output weight $\mathrm{W}_{\mathrm{b}}$ is an $\mathrm{N} \times \mathrm{L}$ matrix which reflects the connection from output nodes to the reservoir. The output of ESN is typically given by

$$
\begin{aligned}
& \mathrm{y}(\mathrm{n})=\mathrm{f}_{\text {out }}\left(\mathrm{W}_{\text {out }} \mathrm{v}(\mathrm{n})\right) \\
& \mathrm{v}(\mathrm{n})=[\mathrm{x}(\mathrm{n}) ; \mathrm{d}(\mathrm{n}-1)]
\end{aligned}
$$




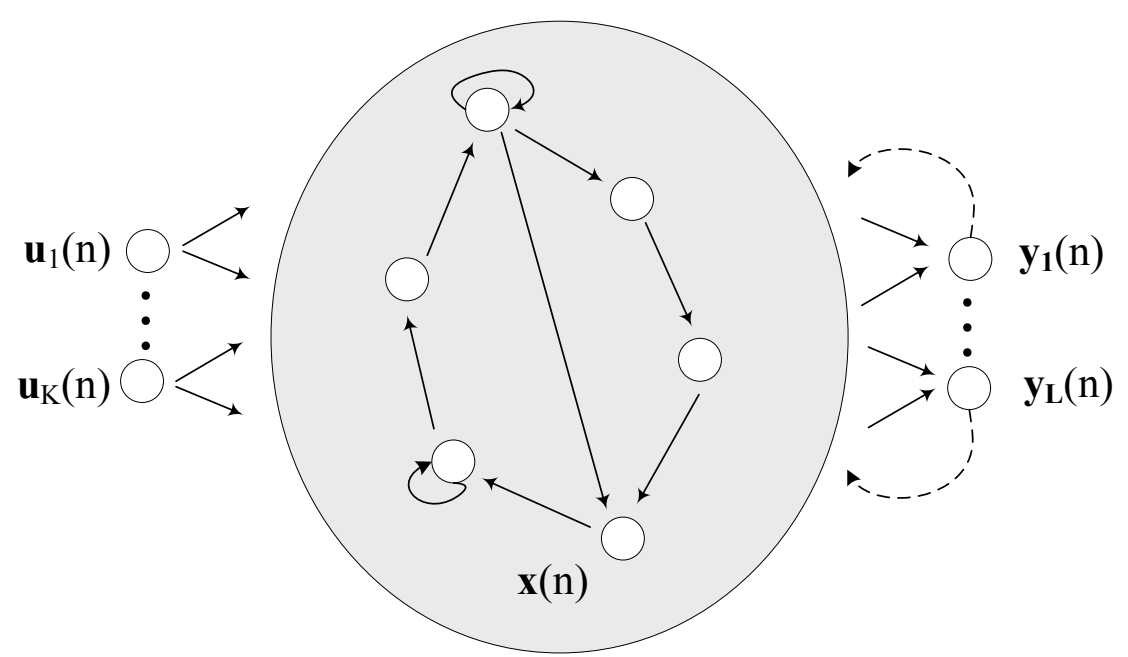

Fig. 1 The basic structure of ESN, with K input node and L output node.

where $f_{\text {out }}$ can be either linear or sigmodal, depending on the task of interest, the output weight $\mathrm{W}_{\text {out }}$ is an $\mathrm{L} \times(\mathrm{K}+\mathrm{N})$ matrix which reflects the connection from the reservoir to output nodes, and $\mathrm{v}(\mathrm{n})$ is the vector of activities of internal state at time step $\mathrm{n}$ and teacher signal at the previous time step. The output weight is determined through either online or offline. In this paper, we use the online RLS for updating output weight and consider one input node and one linear output node. In this way, $\mathrm{W}_{\text {in }}, \mathrm{W}_{\mathrm{b}}$ and $\mathrm{W}_{\text {out }}$ are all vectors.

\section{RLS Algorithm}

ESN training procedure is a simple adjustment of output weight to fit training data. Output weight is updated every time step according to the following equations:

$$
\begin{aligned}
& \mathrm{k}(\mathrm{n})=\mathrm{P}(\mathrm{n}-1) \mathrm{v}(\mathrm{n}) /\left(\mathrm{v}^{\mathrm{T}}(\mathrm{n}) \mathrm{P}(\mathrm{n}-1) \mathrm{v}(\mathrm{n})+\lambda\right) \\
& \mathrm{P}(\mathrm{n})=\lambda^{-1}\left(\mathrm{P}(\mathrm{n}-1)-\mathrm{k}(\mathrm{n}) \mathrm{v}^{\mathrm{T}}(\mathrm{n}) \mathrm{P}(\mathrm{n}-1)\right) \\
& \mathrm{W}_{\text {out }}(\mathrm{n})=\mathrm{W}_{\text {out }}(\mathrm{n}-1)-\mathrm{k}(\mathrm{n})(\mathrm{d}(\mathrm{n})-\mathrm{y}(\mathrm{n}))
\end{aligned}
$$

where $k(n)$ denotes the innovation vector calculated at time step $n$. $d(n)$ and $y(n)$ denote the teacher signal and calculated network output vector, respectively. $\mathrm{P}(\mathrm{n})$ denotes error covariance matrix initialized with large diagonal values and updated at time step n. Forgetting parameter $\lambda>0$ is usually set to the value smaller or equal to 1.0.

\section{Data description}

In this study, the McMaster IPIX datasets are used to be training time series for testing the performances of ESN in detecting target. The McMaster IPIX radar is an instrumentation-quality X-band radar system. The radar data were collected in November 1993 from Osborne Head Gunnery Range (OHGR) at Dartmouth, Nova Scotia, Canada. Fourteen sea clutter measurement datasets were obtained from a website maintained by Professor Simon Haykin: http://soma.ece.mcmaster.ca/ipix/dartmouth/datasets.html. The operating frequency of IPIX radar is $9.39 \mathrm{GHz}$, so the wavelength is about $3 \mathrm{~cm}$. The wave height of the ocean varies from $0.8 \mathrm{~m}$ to $3.8 \mathrm{~m}$, but the peak height even arrives at $5.5 \mathrm{~m}$. The wind conditions vary from 0 to $60 \mathrm{~km} / \mathrm{hr}$ generally, but the gusts reach to $90 \mathrm{~km} / \mathrm{hr}$. The grazing angle varies from less than $1^{\circ}$ to a few degrees. We consider amplitude data of two polarizations, HH (horizontal transmission, horizontal reception) and VV (vertical transmission, vertical reception). Each dataset contains fourteen spatial range bins, and each range bin has $2^{17}$ samples and the sampling frequency is $1000 \mathrm{~Hz}$. The target is a small spherical block 


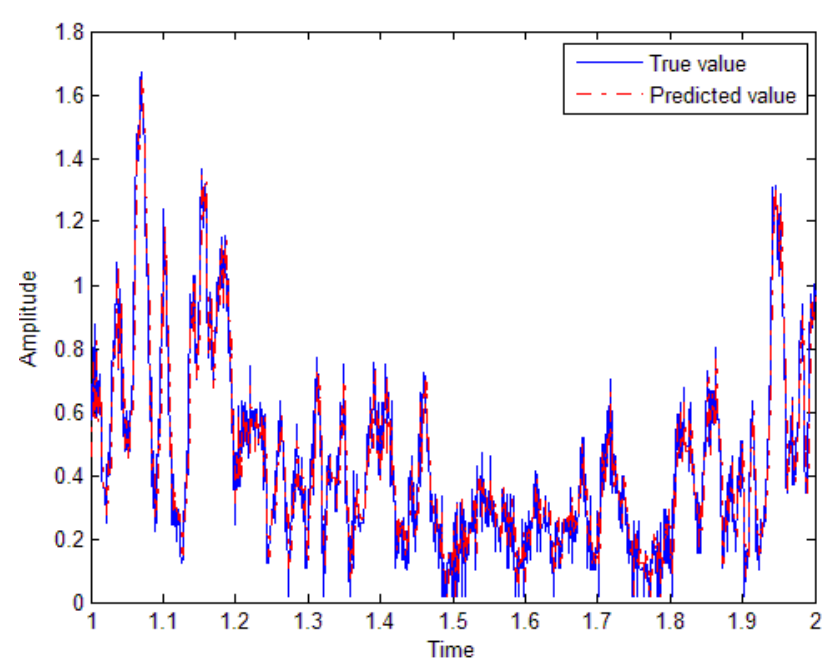

Fig. 2 Prediction curves for the range bin without target.

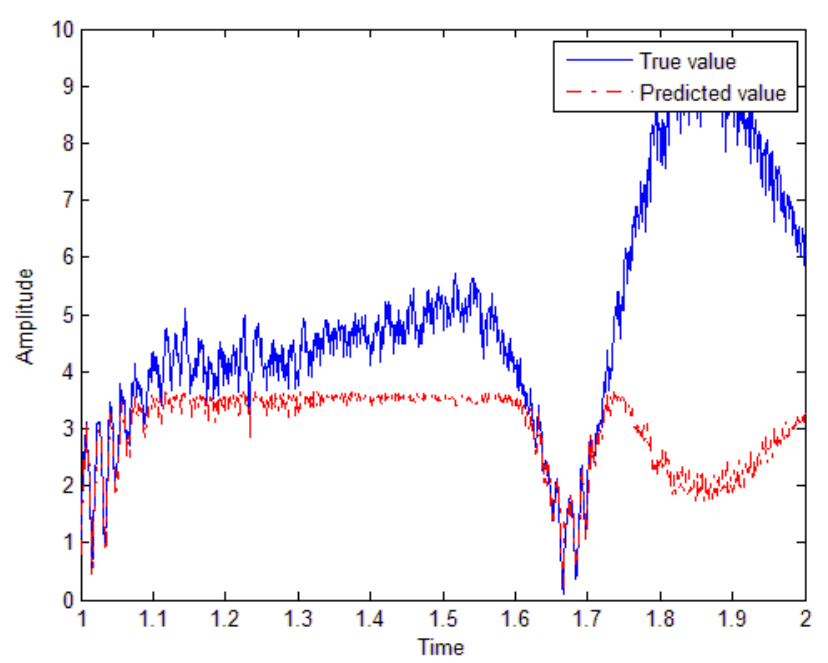

Fig. 3 Prediction curves for the primary target range bin.

of styrofoam wrapped with wire mesh. A few of the range bins hit a target, and the range bin where the target is strongest is labeled as the primary target bin. Due to the target moves around, the bins close to the primary target bin may also hit the target. They are labeled as the secondary target bins.

\section{Experiments}

We study the performance of ESN with IPIX radar sea clutter data. The number of neurons in the dynamic reservoir is 30 . The non-zero entries of Win and Wback are uniformly distributed random variables within the range $[-0.05,0.05]$ and $[-4,4]$, respectively. The entries of the recurrent weight $\mathrm{W}$ are randomly created with a sparsity of $10 \%$ and was rescaled to spectral radius $\rho(W)=0.1$. The entries of the artificial noise which inserted into the state updated equation are random number within the range $[-0.0001,0.0001]$. RLS forgetting parameter $\lambda$ was set to 0.995 .

The typical prediction curves of amplitude versus time for the range bin of sea clutter without target is shown in Fig.2, and the range bin of sea clutter with target is shown in Fig.3. Blue solid line denotes the real-life sea clutter data, and red dashed line denotes the prediction curve of ESN. From Fig.2, we observe that the prediction curve is in good agreement with the real-life sea clutter data, which indicates ESN has high accuracy for predicting sea clutter time series. Since the ultimate goal of sea clutter study is to improve the performance of target detection within clutters. We can use the above ESN method to detect target embedded in sea clutter. MSEs for the fourteen range bins data are shown in Fig.4. It is not difficult to see that MSE for the data with the primary target is much larger than that without the target. It turns out that this is a generic feature for all the measurement data.

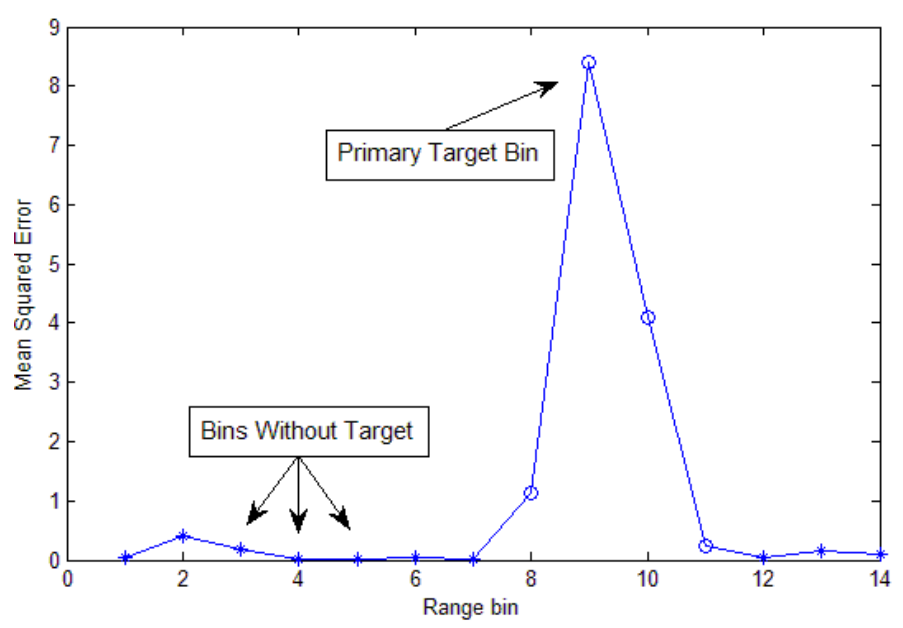

Fig. 4 The mean squared errors for the 14 range bins. 


\section{Summary}

We apply ESN for predicting sea clutter time series. The experiments demonstrate that ESN has higher prediction precision for pure sea clutter data than that with target. This characteristic can be used to detect target effectively.

\section{References}

[1] H. Jaeger, The echo state approach to analysing and training recurrent neural networks. Tech. Rep. Fraunhofer Institute for Autonomous Intelligent Systens: German National Research Center for Information Technology (GMD Report 148) (2001).

[2] H. Jaeger, Adaptive nonlinear system identification with echo state networks. In S. T. S. Becher, \& K. Obermayer (Eds.), Advances in neural information processing systems (pp. 593-600). Cambrige, MA: MIT Press (2003).

[3] H. Jarger and H. Haas, Harnessing nonlinearity: Predicting chaotic systems and saving energy in wireless communication. Science, 304(5667), 78-80 (2004).

[4] K. Ishii, T. van der Zant, V. Becanovic, and P. Ploger, Identification of motion with echo state network. In Proc. OCEANS'04 (2004), p. 1205-1210

[5] P.G. Ploger, A. Arghir, T. Gunther, and R. Hosseiny, Echo state networks for mobile robot modeling and control. In Proc. RoboCup (2003), p. 157-168 